\title{
Author Index Vol. 56, No. 4, 1995
}

Numbers refer only to Abstract numbers

Abdiu,A. 39,40 Adler, G. 41,49 Adrian, T.E. 2, 113, 140 Alfonsi,G. 31 Anastasi, G. 95

Andersson, H. 69 Ando,E. 101 Andreaus, M.C. 143 Andrén-Sandberg, Å. 1, 69 Angelini, G. 28 Angly,B. 65 Antolín,M. 152 Aprahamian, M. 61,147 Arita,Y. 68 Arnelo, U. 2 Arnold, R. 73,128 Ashraf,H. 3 Astier,J.-P. 54

Baczako, K. 47

Baeza, N. 4

Bailey, B. 7

Baize, M. 35

Balagué,C. 145,146

Balestracci, T. 23

Ballé,C. 63

Balzano, G. 5,6, 159

Bank,S. 7

Bannai, S. 125

Barakat,B. 14

Barbe,L. 112

Barbi,G. 89

Bardají, M. 24

Baroncini, D. 14

Barthet,M. 8,82

Bassi,C. 6,108,109,114

Basso, D. 43,104

Bauer, I. 60

Beaux,A.C.de 9,10

Becker, H.D. 12

Beechey-Newman, N. 11

Beger, H.G. 15, 29, 36, 44, 52, 116, 127,

130,132,139 Beglinger, C. 3, 65 Bellini, O. 89 Benz,S. 12 Bergamo, LA. 114 Bernades,P. 82, 112, 119 Bernard, J.P. 8 Berthélémy, P. 103 Bielanski,W. 13 Biemond, I. 78 Billi,P. 14,110 Bilski,J. 13

Birk,D. 15,44,127

Blanco, C. 16

Blanco, I. 123

Bloss,U. 63

Bogina, G. 17

Boistelle, R. 54

Bonora,A. 108, 109

Bordas, J.M. 145,146 
Borsato,N. 107

Boruchowicz, A. 82

Bovo,P. 18,28,143,144

Bradford, I. 98

Bragado,J. 19,20,122

Bramhall, S.R. 21

Bründler,M. 48

Brunori,M.P. 18,28

Büchler, M.W. 29,36,47,48,49,131,151

Buck, A. 15,44

Bulbena,O. 66

Burchard-Reckert, S. 79

Buscail,L. 22,26, 103

Busing, M. 12

Butturini,G. 6,108,109,144

Caballería, J. 102

Calan,L. de 82

Caldiron,E. 108,109,144

Calori, G. 6

Calvo,JJ. 19,20,122

Campani, D. 23

Capella,C. 80

Capellá,G. 37,100,155

Cappelletti, O. 110

Carballo,F. 96

Caroli,A. 137

Carroccio, A. 95

Carter,D.C. 9, 10,38,76

Casarini, M.B. 143,144

Case, R.M. 70

Casellas, F. 152

Caumo, F. 115

Cavallera,A. 88

Cavalhni,G. 17, 18,28,50, 108, 109, 115,

143, 144 Ceccarelli, F. 23 Cecchetti,D. 23 Cellini, N. 30,31 Cengia,C. 121 Ceranowicz, P. 27

Chalmers, A.G. 81 Chalupka,B. 138 Christ, A. 3

Ciambra,M. 88 Cieszkowski, M. 13 Cipolla,A. 154 Clerc,P. 34 Closa,D. 24,66 Coll, A. 25

Cooper, A. 117 Corominas, J.M. 87 Costamagna, G. 30 Cremer, M. 35 Creutzfeldt, W. 42

Crucitti,F. 30,31 Crul,BJ.P. 134 Cuatrecasas, M. 37

Dagorn, J.-C. 54

DalГO',E. 143

Dal Monte, P.R. 14

Damgé, C. 61

D’Angeli,F. 90

D'Angelo,P. 95

Daniel, R. 8 
D'Antonio, L. 23

Dardanoni, G. 88

Deflorin,J. 49

Delacoux, E. 119

Delcenserie, R. 82

Delesque, N. 26

DelFavero, G. 41,90

Delhaye,M. 35

DelMistro,A. 104

Dembiński, A. 27

DePaoli,M. 43,90

DeSanti,S. 80

DeSantis,L. 108,109

Devière, J. 35

Di Carlo, V. 5,6,159

Di Francesco, V. 18,28,143, 144

D'lmperio, N. 14

DiPrima,F. 94

Di Sebastiano, P. 29

Doglietto,G. 30,31

Domínguez-Muñoz, J.E. 32, 33

Domschke,W. 158

Dueñas, C. 64

Dufresne, M. 34

Dumonceau, J.-M. 35

Effert,B. 33 Eisele, S. 36 Elton, R. 38 Emmrich, J. 136 Erill,N. 37,155

Erlanson-Albertsson, C. 91 Escourrou, J. 103 Escrieut, C. 34 Espert,J.J. 145 Estève,J.P. 22,26 Evrard,S. 147

Falconer, J.S. 38

Falconi,M. 6, 108, 109,114,143

Fanjul, M. 34

Farré,A. 37,100,123,155

Fearon, K.C.H. 9, 10, 38, 76

Feng,W. 39,40

Ferlin,G. 107

Fernandez, E. 16

Fernandez, M.D. 64

Fernández-Cruz, L. 24, 66, 102

Fernández-del Castillo, C. 46

Fernandez Forcelledo, J.L. 64

Ferrara,C. 41,90

Figarella, C. 4,97

Filippini, M. 18,28

Fink, T. 29

Fiocchi,M. 110

Fitting, Th. 84 
Fogar, P. 43

Fölsch,U.R. 42,60,84,85,126

Formentini, A. 15, 44

Fornaciari, G. 23

Fourmy, D. 34

Freiburghaus, A.U. 67,135

Frena, A. 45

Frexinos, J. 103

Frick,T.W. 46

Friebe,W.-G. 131

Friess,H. 29,47,48,49,151

Frontera,D. 30,31

Frulloni,L. 18,28,50,143,144

Furuya,S. 101

Gabryelewicz, A. 120

Galloway, S.W. 51,74

Gansauge,F. 15,52,127,139

Gansauge, S. 127

Garcia, C. 155

Garcia, L.J. 19,20,57

Gasslander, T. 40

Gaudio,A. 18

Geada,M.M. 53

Geada,M.R. 133

Gebhard,R. 141

Geider,S. 54

Gelpí, E. 24, 66

Gentilini,O. 5

German Study Group of Acute Biliary

Pancreatitis 42 Giacovelli, G. 94 Giardino,D. 23 Gielkens, H.A.J. 55,78 Giménez, A. 102

Giulianotti, P.C. 23

Glasbrenner, B. 32

Goebell,H. 118

Göke,B. 73,128,129

Gold, L.I. 48

Goldie, A,S. 10

Gonzalez, A. 56, 57

Gracia, X. de 152

Graziani,R. 109,114

Green, N.K. 58

Grendell, J.H. 86

Gress, T. 41

Grönroos, J. 77

Gross, V. 92

Gruppo Italiano Gabesato Endoscopia 
Digestiva 50 Guarise, A. 114 Guarner, L. 152 Guillaumes, S. 123 Gullo,L. 59 Gundlach,F. 60 Günther,R. 60 Guolo,P. 137 Guy-Crotte, O. 97 Gyr,K. 3

Hajri,A. 61,147 Halangk,W. 93 Hammel,P. 119 Han, B. 62, 124 Haunschmid, B. 129

Hausmann, D. 136 Häussinger, D. 62, 75, 86,124 Hayakawa, T. 101 Hegyi,P. 142

Heimburger, N. 12 Heinisch,A. 63,92 Heinmöller, E. 157 Heisel,F. 147 Heras, G. de las 64 Heresbach, D. 82 Hermon-Taylor, J. 11 Herrington, M. 140 Hildebrand, P. 3,65 Hilgers, R.A. 42 Hokenmaier, J. 116 Hollerbach, S. 92 Holstege,A. 92 Hopman, W.P.M. 148, 149

Hopt,U.T. 12 Hotter, G. 24,66 Hotz,H. 67 Hou,W. 68 Huupponen, R. 77

Ihse, I. 1,69,160

Innocenti, P. 29

Ishiguro, H. 70

Ishii,T. 125

Italian Pancreatic Cancer Study Group 59

Jansen, J.B.M.J. 134,148,149

Jármay,K. 142

Jäschke, A. 136

Jenkins, S.A. 156

Jensen, R.T. 57

Jesnowski, R. 83

Johnson, CD. 117

Jones, B.M. 98

Juma, L.M.O. 133

Jurkowska, G. 120

Kadow,R. 63 Kaila,T. 77 Kaiser, A.M. 71,72 Kalthoff, H. 22, 83 Karran,S.J. 117

Katschinski, M. 73,128,129 Kazakoff,K. 113 Kennedy, K. 34 Kerr,DJ. 58 Ketterer,S. 65

Keuper, H. 12 Kingsnorth, A.N. 51,74 Kisker,O. 138,157 Kissling,P. 47 Kitagawa, M. 101

Klar,E. 67

Klonowski,H. 62,124 Klonowski-Stumpe, H. 75 Knust,EJ. 118 Köhler,H. 79 Konturek, S.J. 13,27 Korc,M. 48,49 Kraus, M. 12 Kuweit, P. 128

Lai,P,B.S. 76 Laine,VJ.O. 77 Lam,W.F. 78 Lamers, C.B.H.W. 55, 78 Langa, F. 25 Lankisch, P.G. 79 La Rosa, S. 80 Larsson, J. 2, 39,40,140 Larvin, M. 81 LeBodic,L. 82 Leckström, A. 39 Lemoine, N.R. 21 Le Moine, O. 35 Leone, B.E. 6,80 Lerch, M. 41 Leser,H.-G. 63,92 Levy, P. 82,119 Liebe,S. 83,136 Liessi,G. 107 Ligabue, A. 154 Link,KH. 52 Linseis, A. 92 Lippert, H. 93 Liras, G. 96

332

Author Index

Liu,J. 140 Llorens, R. 16 Lluís,F. 37,100,123,155 LoCasto,A. 95 Lock,G. 92 Löhr,M. 83,136 Lonovics, J. 142 Lopez, J.M. 102 Lopez, M.A. 19,20,122 López-Novoa, J.M. 122 Lorenz, R. 75 Loser, Ch. 84,85 Loualidi,A. 149 Lüdtke,R. 42 Lüthen,R. 75,86 Lutz,M. 41

MacFie,J. 98 McMahon, MJ. 81 Mahmood,A. 98 Mainardi, A.P. 114 Malagelada, J.-R. 152 Malats,N. 87 Malfertheiner, P. 32, 33 Malizia,R. 95 Manes, G. 88 Mann,G.E. 125,150

Mann,S. 63 Mansueto, GC. 115 Marchand,S. 97 Marcori, M. 28 Marescaux, J. 147

Maringhini, A. 88 Marosi, E. 106 Marotta,F. 89 Martinez, E. 64 Martinez, J. 145, 146

Masclee, A.A.M. 55,78 Mattfeld,T. 44 Matthias, R. 93 Mazo, A. 25 Meggiato, T. 90 Mei,J. 91 Meier, R. 3 Melzi d'Eril, G.V. 153 Mengod, G. 34 Merigo, F. 18 Merkord,J. 136 
Messmann, H. 63, 92 Meyer, F. 93 Meyer Wyss, B. 3 Miah,A. 7 Midiri,M. 95 Miglioli,M. 14,110 Militelli,C. 94 Millat,B. 82 Mirmiran-Yazdy, A. 7 Mitchell, C.J. 98 Mithöfer,K. 46 Mittempergher, F. 121

Moi,A. 143 Molas,G. 112 Mölgaard, A. 85 Molina, M.A. 16 Molloy,R,G. 9 Montalto,G. 95 Mooren,Ch. 158 Mora, A. 96 Moratti,R. 153 Moreau, J. 82, 103 Morganti, A.G. 30,31 Moriscot,C. 4,97 Morisset, J. 68 Moroder, L. 34 Morselli-Labate, A.M. 59 Mueller-Pillasch, F. 41 Mühlmeier, G. 132 Müller,M.K. 118 Müller,P. 83 Murchan,P. 98 Mussack, T. 99 Nabau,N. 100 Naccarato, R. 90 Nagy,I. 142 Naruse,S. 101 Navarro,S. 102,145 Nedeleff,B. 93 Neoptolemos, J.P. 21,58 Neumann, P. 126 Nevalainen, T.J. 77 Nicoli,N. 115 Niederau, C. 62, 75, 86,124 Nilsson,C. 160 Nilsson, L. 40 Nitsche,R. 42 Nitsche,S. 54 Nokihara,K. 101 Okamoto, H. 4

Pages, P. 103

PANK-ras I Project Investigators 87

Panozzo, M.P. 43, 104

Pap,Á. 105,106

Pares, A. 102

Pariente, A. 82

Pariente, J.A. 56

Parolini,D. 159

Pascual,S. 145

Pasquali,C. 43,107,137

Patti,R. 88

Pederzoli,P. 6, 17,18,28,108,109,115,

143, 144 Pedrazzoli, S. 94,107,137 Perez Ayuso, R.M. 145,146 Perez-Mateo, M. 96

Permert,J. 2,39,40,140 Petersen, M. 79 Petersen, O.H. 156

Pezzilli, R. 14, 59, 110

Pfeffer,F. 12

Pi,F. 66

Piemontese, A. 14

Piiper,A. Ill,141

Piñol,J.L. 87

Plebani,M. 43,90,104

Pollini,E. 153

Polverosi, R. 137

Pons Romero, F. 64

Ponsot,P. 112

Porta,M. 87

Pour,P,M. 37,113

Pozsár,J. 105

Pradhan,T,K. 57

Procacci,C. 17,28,108,114,115

Pros, I. 146

Rabitti,P.G. 88 Rachid,H. 119 Rae,D. 11 Rattner,D.W. 46 Rau,B. 116 Rauly,I. 26

Ravichandran, D. 117 Real,F,X. 34,87 Redha,F. 67 Rehbehn,K. 118 Reidelberger, R. 2

Reske,S.N. 139 Reyes, G. 155 Rhein,E. 130 Ribera,M.T. 123 Rifà,J. 87 Rigo,L. 18,28

Rindi,G. 80 Robberecht, P. 22 Robinson, P.J. 81 Rodriguez, J. 145 Rodriguez-Nodal, F. 122 
Roermund, R.F.C. van 148 Ros,E. 145,146 Rosado, J.A. 57 Roselló-Catafau, J. 24, 66 Ross,J $\Lambda$. 9,10,38,76 Rosso, A. 159 Rovati,L.C. 94 Roveroni, G. 43 Rünzi,M. 118 Ruszniewski, P. 112,119 Rydzewska,G. 120

Sadowski,Ch. 131 Safi,F. 15,44 Safran,P. 89 Sahel,J. 8 Saillan,C. 34 Saint-Laurent, N. 22 Salerni,B. 121 Salido,GM. 56

Saluja,A. 71

Saluja,M. 71,72

Salvia,R. 6,108,109,144

Sammartano, S. 115

Sanchez, J. 96

Sanchez Antolin, G. 64

San Roman, J.I. 19,20,122

Sans,M.D. 123

Sarles,H. 8

Sartori,N. 108,109,144

Sastre,S. 150

Sata,H. 62

Sata, N. 124

Sato,H. 125

Sauerbruch, T. 32, 33

Sauvanet, A. 82

Scala,L. 88

Schäfer,H. 126

Schäfer,T. 73

Schaller,Ch. 127

Schally,A.V. 26

Scheurer,W. 131

Schild,L. 93

Schilling, M. 47,48

Schirra,J. 73, 128,129

Schirren, A. 79

Schlosser,W. 130

Schmid, R. 49

Schmid,St. 151

Schmidt, J. 63

Schmidt, W.E. 60,126

Schmitter,N. 131

Schoenberg, M.H. 36, 116, 127, 130

Schölmerich, J. 63, 92

Schrag,H.J. 131

Schropp,T. 157

Schulz,H.-U. 93

Searle,P.F. 58

Sengupta, A. 71

Sessa,F. 80

Sgarbi,D. 18 
Siech,M. 132

Singh, J. 53,56,133,158

Slappendel, R. 134

Solcia,E. 80

Soresi,M. 95

Sörhede, M. 91

Soriani,M. 135

Sowinska, M. 147

Sparmann, G. 83, 136

Sperti,C. 43,94,107,137 Spies, Th. 138,157 Staib,L. 139 Stamp, G.W.H. 21 Stark, B. 7 Steer, M.L. 71,72 Steward, M.C. 70 Stöckmann,F. 79 Stoll,R. 158 Stollfuss, J.C. 139 Strömmer, L. 140 Stryjek-Kaminska, D. Ill,141 Stupnicki, A. 151 Sumar,N. 11 Susini,C. 22,26 Sutton, R. 156 Sweiry,J.H. 125,150

Tajiri,H. 89

Takács, T. 142

Taketani,S. 125

Talamini, G. 18,28, 108,109,143,144

Targarona, E.M. 145,146

Tasseti, V. 147

Tebaldi,M. 28

Terés,J. 145,146

Termini, A. 88

Teschauer,W. 99

Tessari, G. 90

Thimister, P.W.L. 148,149

Thomas, F. 61

Tibes,U. 131

Tittobello, A. 50

Tolo, C. 17

Tömösközi, S. 105

Townsend, CM., Jr. 37

Trias, M. 145,146

Trautmann, B. 83

Trauzold,A. 126

Trodella,L. 31

Trowell,S.N. 150

Ubalducci, G.M. 153,154 Uhl,W. 47,48,49,131,151 Uomo, G. 88 Usellini,L. 80

Valderrama, R. 102 Valdo,M. 114 Valentini,V. 30

Vallat,F. 61 Vandermeeren, A. 35 Vaona,B. 18,28,143,144 Vaquero, E. 152 Vaysse, N. 22, 26, 34 Vecht,J. 55 Vela,M. 145,146 Ventrucci,M. 153, 154 Verdier, J.-M. 54 Verheijen, R. 134 Vesentini, S. 108, 109 Vialettes, B. 4 Viedma,J.A. 96 Vilgrain,V. 112 Villa, E. 159 Villanueva, A. 155 Viña,J. 150 Viola, G. 30,31 Vogel,R. 151 Vogt,W. 92 Voitot,H. 119 Völkel,K. 63

Waldner,H. 99 Wank,U. 128,129 Ward,J,B. 81,156 Warshaw, A.L. 46 Warzecha, Z. 27 Weber, A. 47 Weber, H.C. 57 Weidenbach, H. 41 Weidmann, C. 73 Weihe,E. 29 Weinel,RJ. 
138,157 Westermark, P. 2, 39 Willems,J.L. 148,149 Wisdom, D.M. 53,56,133,158 Witt,M. 126 Woestenborghs, R. 148 Wray, V. 101

Young, L.S. 58

Zamboni,G. 17,108,109,114 Zellner,G. 131 Zerbi,A. 5,6,159 Zeuzem, S. Ill,141 Zicari, M. 115 Zirngibl,H. 63 Zoucas, E. 1, 160

334

Author Index 